\title{
Bridging the Atlantic gap in clinical guidelines for non-ST-elevation acute coronary syndromes
}

\author{
Francesco Nudi, MD, ${ }^{\mathrm{a}, \mathrm{b}, \mathrm{c}}$ Alessandro Nudi, $M D,{ }^{\mathrm{c}}$ Giuseppe Biondi-Zoccai, MD, \\ MStat, ${ }^{\mathrm{d}, \mathrm{e}}$ and Orazio Schillaci, $\mathrm{MD}^{\mathrm{e}, \mathrm{f}}$ \\ a Service of Hybrid Cardio Imaging, Madonna Della Fiducia Clinic, Rome, Italy \\ b Ostia Radiologica, Rome, Italy \\ c Etisan, Rome, Italy \\ d Department of Medico-Surgical Sciences and Biotechnologies, Sapienza University of Rome, \\ Rome, Italy \\ e Department of AngioCardioNeurology, IRCCS Neuromed, Pozzilli, Italy \\ f Division of Nuclear Medicine, Tor Vergata University, Rome, Italy
}

Received Jan 15, 2018; accepted Jan 15, 2018

doi: 10.1007/s12350-018-1216-9

\section{See related article, pp. 769-776}

Common sense is judgment without reflection, shared by an entire class, an entire nation, or the entire human race.

Giambattista Vico

\section{PREAMBLE}

American and European clinical practice guidelines for non-ST-elevation acute coronary syndromes (NSTEACS) represent a unique opportunity to analyze in detail and reshape the role of cardiac imaging tests in the modern era of cardiovascular medicine. ${ }^{1,2}$ As it is often the case, differences and gaps should pose a constructive challenge, and prompt readers and practitioners to improve knowledge and decision making. Thus, we must be thankful to Prejean et al. for providing us in this issue of the Journal a detailed comparative analysis summarizing agreements between such guidelines while highlighting important discrepancies. ${ }^{3}$ As in guidelines on stable coronary artery disease (CAD),

Funding This work was supported by Etisan, Rome, Italy.

Reprint requests: Francesco Nudi, MD, Service of Hybrid Cardio Imaging, Madonna della Fiducia Clinic, Via Giuseppe Mantellini 3 , 00179 Rome, Italy; francesco.nudi@gmail.com

J Nucl Cardiol 2018;25:780-4.

$1071-3581 / \$ 34.00$

Copyright (C) 2018 American Society of Nuclear Cardiology. even in NSTEACS guidelines the clinical condition is the starting point which represents, in this case well, a potential complication in the natural history of CAD.

In stable CAD, nuclear cardiology has a key diagnostic and prognostic role, as in patients with a good prognosis, in whom it might seem irrelevant to proceed with revascularization in comparison to optimal medical therapy, myocardial perfusion imaging (MPI) recognizes the group of subjects at higher risk of severe adverse events. ${ }^{4}$ In patients with NSTEACS the management approach is of course altogether different, but discrepancies are evident also on the diagnostic approach. ${ }^{1-3}$ Indeed, in NSTEACS, at odds with stable CAD, pre-test probability of CAD receives much lesser emphasis. The first issue rests on goals of tests and key limitations of test results. While for stable CAD guidelines the key weakness of tests relies in false positives, in NSTEACS it rests on false negatives, especially given the medico-legal liability implications. Another important feature is appraising the relationship among diagnostic tests which differ in role, in particular stress ECG, functional imaging tests (echocardiography, cardiac magnetic resonance [CMR], and myocardial perfusion imaging $[\mathrm{MPI}]$ ), and anatomic imaging tests (coronary computed tomography [CT]). It is also important to distinguish functional tests based on the use of a dynamic vs pharmacologic stress.

\section{INVASIVE STRATEGY}

Patient heterogeneity in coronary anatomy in NSTEACS is substantial, with many subjects lacking significant coronary lesions. ${ }^{5}$ Conversely, multivessel 
disease may have a prevalence ranging between $40 \%$ and $80 \%$. Accordingly, unless very high-risk features mandating an immediate invasive strategy are present, a rule-in diagnosis is best sought in the emergency room (ER) or in the chest pain unit (CPU), given the multitude of causes of chest pain. ${ }^{1,2}$ Such approach is based on the triad of symptoms, ECG changes, and high-sensitivity troponin (hsT). In particular, symptoms may vary or lack altogether, even if when present the dominant type is prolonged (> 20 minutes) chest pain at rest $(80 \%)$, followed by new-onset chest pain (10\%), and worsening or post-myocardial infarction (MI) chest pain (10\%). Typical ECG changes include transient ST elevation, persistent or transient ST depression (occasionally specular), negative or pseudonormalized $\mathrm{T}$ waves. ${ }^{1,2}$ Notably, ECG may be apparently normal in $30 \%$ of subjects, with left bundle branch block posing major diagnostic issues. While hsT has a high negative predictive value for ruling out MI, several conditions may cause an increase in hsT associated with chest pain, complicating the differential diagnosis. Accordingly, it is useful to evaluate the absolute value of hsT, especially when very high, given its strong positive predictive value for ruling in MI, in as much as $75 \%-80 \%$ of patients. In such subjects a default invasive approach is better than a selectively invasive one for most endpoints, including repeat hospitalizations and revascularizations after discharge, but timing is still debated. Indeed, while the overall rate of major adverse cardiac events (MACE) is similar in an early vs delayed invasive strategy, riskstratification using clinical risk scores is useful to identify subjects who can gain a reduction in MACE risk with an early $(<24$ hours $)$ invasive management (i.e., those at high risk in comparison to those at moderate risk). The final decision on management strategy rests on both physician and patient, based on an accurate risk-benefit balance.

Imaging for patients with suspected or established NSTEACS remains crucial in different clinical and temporal scenarios, in particular in the acute phase, during the hospitalization, and after discharge. ${ }^{1-3}$

\section{ROLE OF IMAGING AT ADMISSION}

The acute phase is clearly characterized by patient heterogeneity, including clinical features. ${ }^{1,2}$ Among subjects acutely presenting with suspected NSTEACS, chest pain may be caused by non-coronary cardiac conditions in $15 \%$, and non-cardiac conditions in $50 \%$. Accordingly, the role of imaging is paramount, when findings from symptoms, ECG, or markers are not explicitly in favor of a default invasive strategy. Even if patients have no coronary instability, they might still have stable $\mathrm{CAD}$ on top of a non-coronary or non- cardiac cause of chest pain, benefiting from early diagnosis and risk-stratification for subsequent management, as well as differential diagnosis. Imaging is not indicated in patients at high or very high risk (for whom it is necessary, respectively, an early or an immediate invasive strategy), with the exception of echocardiography to exclude mechanical complications according to European guidelines. ${ }^{1-3}$ Instead, imaging is crucial in particular for low-risk patients in whom a selective invasive strategy is envisioned. Imaging may also be beneficial in those subjects who undergo revascularization within 72 hours from symptom onset but not acutely. These are intermediate risk subjects, who include several clinical conditions, in particular: 1) for diabetics and those with renal failure it is important to appraise the extent and severity of myocardial ischemia; 2) in patients with reduced ejection fraction it is paramount to recognize myocardial viability, contractile reserve, and dyssynchrony; 3) in subjects with prior revascularization it is crucial to evaluate ipsi- or contralateral ischemia, especially in case of incomplete revascularization; and 4) in patients with prior evidence of myocardial ischemia at imaging, it is paramount to appraise defect progression. For these reasons, stress imaging tests are preferred to stress ECG tests. Other indications pertain to the appraisal of possible neuronal injury, for instance, in subjects with significant ventricular arrhythmias. Furthermore, a rest imaging test can occasionally be performed as a stand alone.

In patients in whom a delayed invasive strategy is probable, echocardiography and CMR are useful in the evaluation of myocardial viability as an alternative to MPI, using myocardial wall thickness and fibrosis as markers. ${ }^{1,2}$ Radioprotection issues are also relevant for hospital personnel and materials if invasive procedures or intensive patient management follow shortly MPI.

\section{ROLE DURING HOSPITAL STAY AND AFTER DISCHARGE}

Since accessing the ER or CPU and throughout, imaging tests remain pivotal to establish short and longterm risk. ${ }^{1,2}$ Higher risk patients are admitted for invasive management and revascularization. In other subjects, it is necessary to identify the best treatment spanning between delayed invasive and discharge with optimal medical therapy only. When revascularization is envisioned, it is paramount to distinguish the role of percutaneous coronary interventions vs coronary artery bypass grafting. For instance, in subjects in whom revascularization is indicated, it is useful to identify the culprit lesion or, conversely, the lack of benefit subsequent to treating a lesion associated with necrotic myocardium, especially in patients with prior or recent 
MI. Another pro of imaging tests rests on the functional appraisal of coronary collaterals, as concomitant nonculprit or culprit occlusions are present in up to $25 \%$ of NSTEACS. In addition, two-thirds of these patients have coronary collaterals, with a variable degree of ischemia.

Even after revascularization for NSTEACS or before discharge, imaging can provide details on reverse remodeling and highlight patients with moderately or severely reduced left ventricular systolic function, who might need an implantable cardioverter defibrillator (ICD) or cardiac resynchronization therapy (CRT).

After discharge for a suspected or established NSTEACS, progression of CAD remains a major cause of morbidity and mortality. ${ }^{1,2}$ Accordingly, even after an apparently successful management of NSTEACS, cardiac imaging tests are important to diagnose and riskstratify disease progression. General recommendations apply to all patients, of course, but specific subgroups of patients may require more attentive management because of heightened risk or more complex differential diagnosis, such as the elderly, women, subjects with multivessel disease, acute or chronic heart failure, diabetes mellitus, renal failure, peripheral artery disease, or comorbidities. The reviewed guidelines and Prejean et al. offer important insights on such populations. ${ }^{1,2}$ Yet, whenever prognostic accuracy is important, such as in high-risk patients both acutely as well as at discharge, the functional details become the major player. Indeed, the higher the patient risk, the greater the need to upscale diagnostic alternatives. Finally, pharmacologic stress testing remains crucial during hospital stay, whereas exercise stress testing is very useful before discharge.

\section{TESTING ALTERNATIVES}

Stress ECG, while commonplace and affordable, is inadequate to stratify patients with NSTEACS at low or moderate risk and is fraught by a substantial likelihood of false-negative results, as well as false-positive ones, in terms of diagnostic yield, but even worse of prognostic yield, notwithstanding the poor or absent correlation between test results and culprit lesion, disease extent, left ventricular geometry, function, and viability. $^{1,2}$ Conversely, stress ECG rests strong on easier interpretation, and does not require extensive training nor expensive equipment. For instance, MPI scoring systems enable a more detailed characterization of the patient, but are more demanding. ${ }^{6}$ Some differences regarding the use of diagnostic tests between American and European guidelines can be explained by the fact that access to invasive angiography is easier and more proactive in the US, resulting in fewer noninvasive imaging tests. ${ }^{3}$ The same explanation may apply to the stronger recommendations favoring CT and anatomic details in American guidelines. Indeed, despite the established fact that on average only half of angiographically significant coronary stenoses cause ischemia, myocardial ischemia is not chosen as the key decisional criterion. ${ }^{7}$

As malpractice and financial issues are substantial, unless expedited invasive management is chosen, an imaging test is indeed useful already before admission to decide whether to actually admit the patient or discharge him/her after temporary observation, as well as to minimize the risk of sudden death or MI after discharge. For this purpose, a CT scan and/or a cadmium-zinctelluride/CT camera should be available to all centers given the limited dimensions and ease of exam. ${ }^{8}$ Indeed, as alternatives to MPI, CT, echocardiography, and cardiac magnetic resonance (CMR) are all appealing. Specifically, CT focuses on anatomic detail and thus it best maximizes negative predictive value, minimizing useless admissions. ${ }^{9}$ Our goal of accurately characterizing each patient has however improved with perfusion CT and fractional flow reserve (FFR)-CT, despite this being often at odds with anatomic CT, as even anatomically significant lesions may be associated with normal FFR values. ${ }^{10}$ This clearly reinforces the importance of the functional detail, despite the fact that FFR validity has been questioned in the acute phase of myocardial injury. Several other limitations to CT apply, including protocol issues, variability in interpretation, need for an expert reader, coronary calcium, prior stenting, heart rate, arrhythmias, and so forth.

Echocardiography still rests strong given its noninvasiveness and lack of radiation exposure, access, ease of use, as well as plethora of additional details such as non-coronary findings and complications. In addition, left ventricular geometry and function, whose prognostic role is unparalleled, is finely appraised by echocardiography. ${ }^{11}$ Finally, CMR is very potent to appraise cardiac geometry and function, but less accessible and still limited by lack of coronary details. ${ }^{12}$

Myocardial perfusion imaging (MPI) remains a powerful test in NSTEACS for its ease, access, reliability, and thus may maintain a dominating role among the available cardiac imaging tests. ${ }^{1,2,13-15}$ Yet, virtually all institutions have no 24/7 access to MPI. In addition, in very high- and high-risk patients, MPI is not useful for diagnostic purposes or short-term prognosis as diagnosis is already quite established and accordingly the indication to invasive management. The main contribution of MPI in this setting rests on viability, but this is typically affected later (as it applies to neuronal damage), unless the admission is for reinfarction rather than de novo NSTEACS. In addition, neuronal injury (informing on prognosis and benefits of beta-blocker therapy and ICD) 
can be measured with meta-iodo-benzyl-guanidine (MIBG) tests. ${ }^{16}$ Overall, the main advantage of MPI is that it provides with a one-stop-shop approach a comprehensive set of functional results with a wide spectrum of characterizing features to best define patient prognosis. Yet, specific settings where MPI may be particularly advantageous include patients with reduced systolic function and limited viability or hibernation/ stunning, left ventricular dyssynchrony, or myocardial dysinnervation/denervation.

Irrespective of the choice of its core components, anatomo-functional hybrid imaging appears particularly appealing for patients with NSTEACS, given their high risk of adverse events during follow-up ( $>10 \%$ 1-year MACE rate). ${ }^{4,7}$ For instance, CT-derived anatomic imaging, which provides for instance details on lesion location, plaque features, vessel remodeling, calcium score, and stenosis severity can be seamlessly combined with MPI-derived functional imaging, which yields several functional parameters provided by MPI, including, most recently, coronary flow reserve. ${ }^{6,13-15}$ Thus, hybrid imaging provides many important results which are complementarily associated with pre-test and stress features, enabling the best clustering of the patient into a low-risk group, or otherwise prompting expedited invasive assessment, or guiding postdischarge management and follow-up.

\section{FUTURE PERSPECTIVES AND CONCLUSIONS}

American and European guidelines provide specific guidance for most patients, from bread-and-butter cases to special cases such as the very elderly. ${ }^{1,2,15}$ Yet, individualized management requires also careful and proactive follow-up. Indeed, the importance of the quest for optimal care shortly and long-after discharge for an otherwise correctly managed NSTEACS is proven by ongoing efforts at improving and streamlining patient empowerment, interactive follow-up, and patient-physician communication with novel information technology platforms such as the Recipe tools developed by Replycare (Rome, Italy). ${ }^{17}$

In conclusion, the main goal of any guideline is its application to the single patient. American and European guidelines on NSTEACS offer important and constructive insights to practising cardiovascular specialists. Imaging specialists remain key in capitalizing agreements and resolving discrepancies.

\section{Disclosure}

Dr. Biondi-Zoccai has consulted by Abbott Vascular and Bayer. Drs. F. Nudi, A. Nudi and Schillaci have nothing to disclose.

\section{References}

1. Amsterdam EA, Wenger NK, Brindis RG, Casey DE Jr, Ganiats TG, Holmes DR Jr, Jaffe AS, Jneid H, Kelly RF, Kontos MC, Levine GN, Liebson PR, Mukherjee D, Peterson ED, Sabatine MS, Smalling RW, Zieman SJ. 2014 AHA/ACC Guideline for the management of patients with Non-ST-elevation acute coronary syndromes: a report of the American College of Cardiology/ American Heart Association Task Force on Practice Guidelines. J Am Coll Cardiol 2014;64:e139-228.

2. Roffi M, Patrono C, Collet JP, Mueller C, Valgimigli M, Andreotti $\mathrm{F}$, et al ESC Guidelines for the management of acute coronary syndromes in patients presenting without persistent ST-segment elevation: Task Force for the Management of Acute Coronary Syndromes in Patients Presenting without Persistent ST-Segment Elevation of the European Society of Cardiology (ESC). Eur Heart J 2015;2016:267-315.

3. Prejean SP, Din M, Reyes E, Hage FG. Guidelines in review: comparison of the AHA/ACC Guideline for the Management of Patients With Non-ST-Elevation Acute Coronary Syndromes and the 2015 ESC Guidelines for the Management of Acute coronary Syndromes in Patients Presenting Without Persistent ST-Segment Elevation. J Nucl Cardiol 2014. https://doi.org/10.1007/s12350017-1137-z.

4. Matsuo S, Nakajima K, Okuda K, Kinuya S. The relationship between stress-induced myocardial ischemia and coronary artery atherosclerosis measured by hybrid SPECT/CT camera. Ann Nucl Med. 2011;25:650-6.

5. Meuwissen M, van der Wal AC, Koch KT, van der Loos CM, Chamuleau SA, Teeling P, de Winter RJ, Tijssen JG, Becker AE, Piek JJ. Association between complex coronary artery stenosis and unstable angina and the extent of plaque inflammation. Am J Med 2003;114:521-7.

6. Nudi F, Pinto A, Procaccini E, Neri G, Vetere M, Tomai F, Gaspardone A, Biondi-Zoccai G, Schillaci O. A novel clinically relevant segmentation method and corresponding maximal ischemia score to risk-stratify patients undergoing myocardial perfusion scintigraphy. J Nucl Cardiol 2014;21:807-18.

7. Novara M, D'Ascenzo F, Gonella A, Bollati M, Biondi-Zoccai G, Moretti C, Omedè P, Sciuto F, Sheiban I, Gaita F. Changing of SYNTAX score performing fractional flow reserve in multivessel coronary artery disease. J Cardiovasc Med (Hagerstown) 2012;13:368-75.

8. Nudi F, Iskandrian AE, Schillaci O, Peruzzi M, Frati G, BiondiZoccai G. Diagnostic accuracy of myocardial perfusion imaging with CZT technology: systemic review and meta-analysis of comparison with invasive coronary angiography. JACC Cardiovasc Imaging 2017;10:787-94.

9. Nudi F, Lotrionte M, Biasucci LM, Peruzzi M, Marullo AG, Frati G, Valenti V, Giordano A, Biondi-Zoccai G. Comparative safety and effectiveness of coronary computed tomography: systematic review and meta-analysis including 11 randomized controlled trials and 19,957 patients. Int J Cardiol 2016;222:352-8.

10. Nakanishi R, Matsumoto S, Alani A, Li D, Kitslaar PH, Broersen A, Koo BK, Min JK, Budoff MJ. Diagnostic performance of transluminal attenuation gradient and fractional flow reserve by coronary computed tomographic angiography (FFR(CT)) compared to invasive FFR: a sub-group analysis from the DISCOVERFLOW and DeFACTO studies. Int $J$ Cardiovasc Imaging 2015;31:1251-9.

11. Cullen MW, Geske JB, Anavekar NS, Askew JW 3rd, Lewis BR, Oh JK. Handheld echocardiography during hospitalization for acute myocardial infarction. Clin Cardiol 2017;40:993-9. 
12. Panovský R, Borová J, Pleva M, Feitová V, Novotný P, Kincl V, Holeček T, Meluzín J, Sochor O, Štěpánová R. The unique value of cardiovascular magnetic resonance in patients with suspected acute coronary syndrome and culprit-free coronary angiograms. BMC Cardiovasc Disord 2017;17:170.

13. Nudi F, Schillaci O, Neri G, Pinto A, Procaccini E, Vetere M, Frati G, Tomai F, Biondi-Zoccai G. Prognostic impact of location and extent of vessel-related ischemia at myocardial perfusion scintigraphy in patients with or at risk for coronary artery disease. J Nucl Cardiol 2016;23:274-84.

14. Nudi F, Biondi-Zoccai G, Schillaci O, di Belardino N, Versaci F, Nudi A, Pinto A, Neri G, Procaccini E, Frati G, Iskandrian AE. Prognostic accuracy of myocardial perfusion imaging in octogenarians. J Nucl Cardiol 2017. https://doi.org/10.1007/s12 350-017-1102-x.

15. Nudi F, Biondi-Zoccai G. Cadmium-zinc-telluride myocardial perfusion imaging: the dream of a single test gets nearer. J Nucl Cardiol 2017. https://doi.org/10.1007/s12350-017-0833-z.

16. Vauchot $\mathrm{F}$, Ben Bouallègue $\mathrm{F}$, Hedon $\mathrm{C}$, Piot $\mathrm{C}$, Roubille $\mathrm{F}$, Mariano-Goulart D. Assessment of the area at risk after acute myocardial infarction using 123I-MIBG SPECT: comparison with the angiographic APPROACH-score. J Nucl Cardiol 2016. https://doi.org/10.1007/s12350-016-0644-7.

17. Chwyl B, Chung AG, Shafiee MJ, Yongji Fu, Wong A. A. deep predict: a deep predictive intelligence platform for patient monitoring. Conf Proc IEEE Eng Med Biol Soc 2017;2017:4309-12. 